Marina Encheva*, Nicole Krüger, Vasil Zagorov, Plamena Zlatkova und Gabriela Angelova

\title{
Digitalisierungsprojekt Bibliografie der Wiedergeburt Bulgariens in Sofia
}

https://doi.org/10.1515/bfp-2020-2027

Zusammenfassung: In der Zeit der nationalen Wiedergeburt Bulgariens im 19. Jh. wurden seit dem Beginn der ausländischen Besetzung durch das Osmanische Reich erstmals gedruckte Bücher in bulgarischer Sprache veröffentlicht. Aufgrund eines generellen Verbots von Druckerpressen in den bulgarisch-sprachigen Gebieten zur damaligen Zeit wurden die Schriften in einem weitläufigen geografischen Raum gedruckt, der von Moskau bis nach New York reichte. Im Projekt „Digitalization of the repertoire of books from the Bulgarian Revival (1801-1878)“, gefördert durch den Bulgarischen wissenschaftlichen Nationalfond, wurden die Werke dieser Periode nun erstmals in einer Online-Bibliografie vollständig nachgewiesen und mit den vorhandenen Volltext-Digitalisaten aus den verschiedenen Bibliotheken und Museen Bulgariens verknüpft.

Die im Rahmen des Projekts entwickelte Webseite bietet zahlreiche Sucheinstiege und Filter, eingebettete Tastaturen für arabische, kyrillische, griechische und lateinische Schrift sowie ein geografisches Informationssystem mit Visualisierungen und einem topografischen Index.

Schlagwörter: Bulgarien; Nationale Wiedergeburt; 18011878; Online-Bibliografie; Digitalisierung; geografisches Informationssystem

\section{Digitalization of the Bibliography of Books from the Bulgarian Revival in Sofia}

Abstract: During Bulgaria's national Revival in the 19th century, books were published in Bulgarian language for the first time since the beginning of the foreign occupation by the Ottoman Empire. Due to a general ban on printing presses in the Bulgarian speaking areas at the time, the books were printed in a huge geographical area, reaching from Moscow to New York.

\footnotetext{
*Kontaktperson: Marina Encheva, m.encheva@unibit.bg Nicole Krüger, nicole.krueger@zhaw.ch Vasil Zagorov, v.zagorov@unibit.bg Plamena Zlatkova, p.zlatkova@unibit.bg Gabriela Angelova,g.angelova@unibit.bg
}

With the project "Digitalization of the repertoire of books from the Bulgarian Revival (1801-1878)", funded by the Bulgarian National Science Fund, the books of this period could be compiled in one comprehensive online bibliography for the first time. The entries are linked with the existing digitized full-texts of the various Bulgarian libraries and museums. The website developed within the project, offers various search filters, embedded keyboards for Arabic, Cyrillic, Greek, and Latin script, as well as a geographic information system with visualizations and a topographic index.

Keywords: Revival books; Bulgaria; online bibliography; 1801-1878; digitalization; geographic information system

\section{Einleitung}

Über bulgarische Bibliotheken und ihre Projekte wurde seit dem EU-Beitritt Bulgariens am 1.1.2007 wenig in der bibliothekarischen Fachliteratur in Deutschland veröffentlicht. Zuletzt gab Grashkina (2007) einen historischen Überblick über die Lage der Bibliotheken in Bulgarien und ihre Geschichte. Sie berichtet ausgehend von der Entstehung der ersten bulgarischen Zaren- und Kloster-Bibliotheken im 9. Jh. ${ }^{1}$ und endet mit der Situation im postsozialistischen Bulgarien und unmittelbar vor dem EU-Beitritt.

Heute nun kommt ein bibliothekarisches Projekt zum Abschluss, das es lohnt, den Blick noch einmal auf Bulgarien zu lenken: Die Digitalisierung der Bibliografie der Wiedergeburt Bulgariens mit der zeitlichen Abdeckung von 1801 bis 1878, dem Jahr der Staatsgründung Bulgariens. ${ }^{2}$

Im Projekt „Digitalization of the repertoire of books from the Bulgarian Revival (1801-1878)“3 galt es, die ge-

$1 \mathrm{Zu}$ dieser Zeit wurde die auf die Brüder Kyrill und Method zurückgehende kyrillische Schrift durch Zar Boris I. von Bulgarien eingeführt und kirchliche Liturgien erstmals auf Bulgarisch anstatt auf Griechisch oder Latein abgehalten. Härtel und Schönfeld (1998) 32. Bulgarien war damit das erste Land, das die kyrillische Schrift einsetzte, die heute u. a. in Bulgarien, Russland, Serbien, der Ukraine, Nordmazedonien und Belarus verwendet wird.

2 http://books.unibit.bg.

3 Das Projekt DN 05/2 from 14.12.2016 wird durch den „Scientific Research Fund at the Ministry of Education and Science, Republic of 


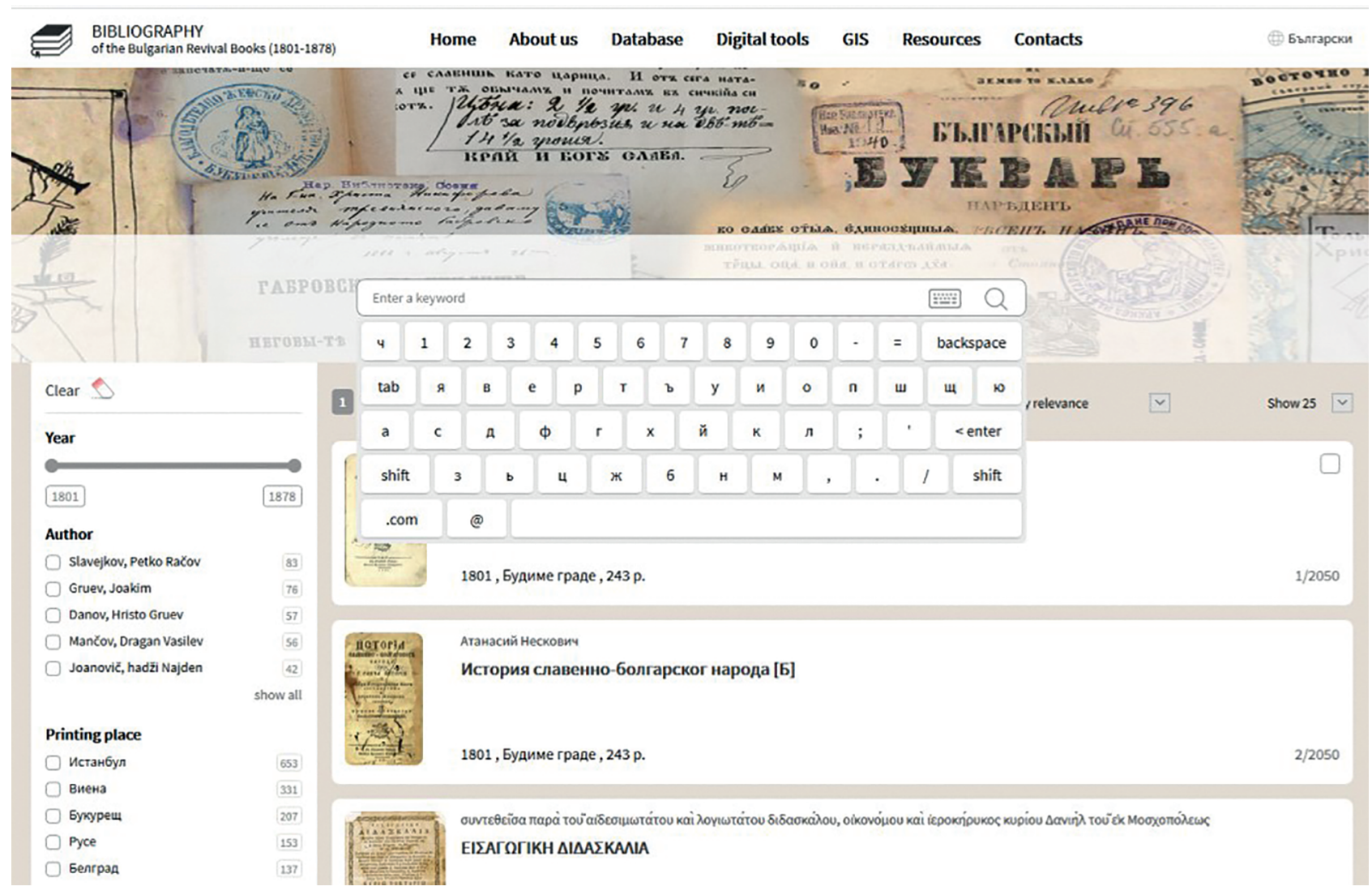

Abb. 1: Suchoberfläche der Online-Bibliografie

samte Literatur dieser Periode zusammenzuführen und zu normieren. Ziel war es zunächst, eine vollständige OnlineBibliografie zu erstellen, um im zweiten Schritt die bereits existierenden Volltext-Digitalisate der verschiedenen Bibliotheken, Archive und Museen Bulgariens zu ermitteln und in der Online-Bibliografie zu verlinken.

Die im Rahmen des Projekts entwickelte Webseite ${ }^{4}$ bietet verschiedene Sucheinstiege, eingebettete Tastaturen für arabische, kyrillische, griechische und lateinische Schrift sowie ein geografisches Informationssystem mit Visualisierungen und einem topografischen Index.

Bulgaria“ gefördert. Es wird durchgeführt vom „Department of Library Sciences at the University of Library Studies and Information Technologies" (ULSIT), Sofia in Zusammenarbeit mit der bulgarischen Nationalbibliothek St. St. Cyril and Methodius. Nähere Informationen zum Projekt auch in Zagorov und Encheva (2017) und Zlatkova und Angelova (2019).

4 http://books.unibit.bg.

\section{Kurzer Abriss zum geschichtlichen Hintergrund der Bibliografie}

Um die Bedeutung der Bibliografie z. B. für die geisteswissenschaftliche oder auch die buchwissenschaftliche Forschung $\mathrm{zu}$ verstehen, ist es wichtig, ein wenig über die geschichtlichen Hintergründe zu erfahren.

Bulgarien war 500 Jahre lang, vom 14. bis ins 19. Jh. Teil des Osmanischen Reiches. ${ }^{5}$ Mit Ausgang des 18. Jh. wurde im Bulgarien der oberen Schichten vermehrt Griechisch gesprochen. Dieses hatte sich als lingua franca, als Umgangs- und Handelssprache auf dem Balkan durchgesetzt. ${ }^{6}$ Teilweise finden sich aus dieser Zeit bulgarische Schriften in griechischer statt in kyrillischer Schrift. ${ }^{7}$ Auch die Schulbildung fand ausschließlich auf Griechisch statt. $^{8}$

5 Crampton (2007) 18.

6 Härtel und Schönfeld (1998) 90.

7 Ebd.

8 Crampton (2007) 49. 
Das Betreiben von Druckpressen war während der osmanischen Herrschaft in ganz Bulgarien verboten. ${ }^{9}$

$\mathrm{Ab} 1800$ kam es, auch angeregt durch nationale Bewegungen in anderen europäischen Ländern, zu einer Wende. ${ }^{10}$ Eine Periode der kulturellen und nationalen Wiedergeburt setzt ein, die auch als bulgarische Renaissance oder National Revival bezeichnet wird. ${ }^{11}$ Ausgehend von den bulgarischen Klöstern erstarken zunächst das Bildungswesen, das Publikationswesen und die bulgarische Literatur. Letztere wird aufgrund der politischen Situation überwiegend im benachbarten oder westlichen Ausland sowie in Moskau gedruckt. ${ }^{12} 1806$ veröffentlicht Ivan Bogorov die erste Schrift in zeitgenössischer bulgarischer Sprache, eine Sammlung von Predigten - gedruckt in Bukarest. ${ }^{13}$ Erste wissenschaftliche Bibliotheken und öffentliche Lesehallen ${ }^{14}$ werden gegründet. 1824 erscheint das erste Schulbuch in bulgarischer Sprache. ${ }^{15}$

Der von Charles Jelavich geprägte Begriff „,bulgarische Inkunabeln" veranschaulicht die Besonderheiten des bulgarischen Wiedergeburtsbuches, das bis zur Mitte des 19. Jh. technologisch und visuell näher an den literarischen Kulturmustern der Gutenberg-Epoche liegt als an dem Niveau des zeitgleichen Buchdrucks in Mittel- und Westeuropa. ${ }^{16}$

Laut Teodosiev entstehen in der Zeit der Wiedergeburt 2227 Titel in bulgarischer Sprache. ${ }^{17}$ Davon sind $91 \mathrm{sog}$. bulgarische „Inkunabeln““ ${ }^{18} 98$ sind Zeitungen aus dem Zeitraum 1806-1878 ${ }^{19}$ und 2038 sind Bücher aus dem Zeitraum 1806-1878. ${ }^{20}$ Diese Daten konnten im Rahmen des hier vorliegenden Projekts aufgrund der Entdeckung von neuen Büchern (66 Titel für den Zeitraum 1801-1878) und der Beseitigung von doppelten Titeln korrigiert und aktualisiert werden.

9 Ebd. 56.

10 Härtel und Schönfeld (1998) 88.

11 Bulg. Българско национално възраждане / bulgarsko nazionalno vasraždane.

12 Crampton (2007) 58.

13 Crampton (2007) 57.

14 Bulg. читалиште/čitalište.

15 Crampton (2007) 55.

16 Jelavich (1957).

17 Teodosiev (2007) 9.

18 Ebd. 56.

19 Ebd. $413 \mathrm{ff}$.

20 Ebd. 9, 56, $413 \mathrm{ff}$.

\section{Projekt-Abschnitt I: Die Online-Bibliografie der Literatur zur Wiedergeburt Bulgariens (1801-1878)}

Das Projekt startete ausgehend von der Situation, dass existierende Versionen der Bibliografie der Wiedergeburt Bulgariens ausschließlich gedruckt vorlagen und sich sowohl in Struktur (sie waren z. B. teilweise alphabetisch, teilweise chronologisch angeordnet) sowie in Menge und Inhalt der nachgewiesenen Titel unterschieden. Eine weitere Schwierigkeit bestand darin, dass das bulgarische Buch des 19. Jh. bibliografisch schwer zu erfassen ist, da:

- der Buchdruck in Bulgarien in dieser Zeit eine rasche und komprimierte historische Entwicklung durchläuft,

- es eine große geografische Streuung der Druckorte gibt,

- sprachliche Besonderheiten den Abgleich von Bibliografien erschweren und

- die zeitlichen und geografischen Grenzen für die Bibliografie schwer festzusetzen sind, weil Bulgarien zu dieser Zeit in nicht festgelegten Grenzen existiert und die zeitlichen Übergänge fließend sind. ${ }^{21}$

Ziel des Projektes war es einerseits, erstmalig einen Online-Zugang zur Bibliografie zu schaffen und diese über Suchmaschinen zugänglich zu machen..$^{22}$ Hierfür mussten die gedruckten Bibliografien zunächst elektronisch erfasst und mit dem Online-Katalog der bulgarischen Nationalbibliothek „St. St. Cyril and Methodius“ sowie weiterer Bibliotheken für den Zeitraum abgeglichen werden. Andererseits sollten bestehende Mängel der gedruckten Bibliografien behoben werden: So sollte in der bereitgestellten Suchoberfläche eine größtmögliche Anzahl von Sucheinstiegen und Sortierkriterien für den Bestand angeboten werden.

Die Online-Bibliografie verfügt aufgrund der druckgeschichtlichen und historischen Besonderheit der Publikationen nicht nur über Informationen $\mathrm{zu}$ Titel und $\mathrm{Au}$ tor`innen sowie Verlagsdaten (Reihenfolge und Merkmale der Ausgabe, Erscheinungsort, Erscheinungsjahr, Druckerei), sondern weist z.B. auch physische Merkmale und

21 Vgl. zu allen vier Punkten Abschnitt (1) zum geschichtlichen Hintergrund.

22 In einem weiteren Schritt sollen die Metadaten als Linked Open Data im Semantic Web veröffentlicht werden. 


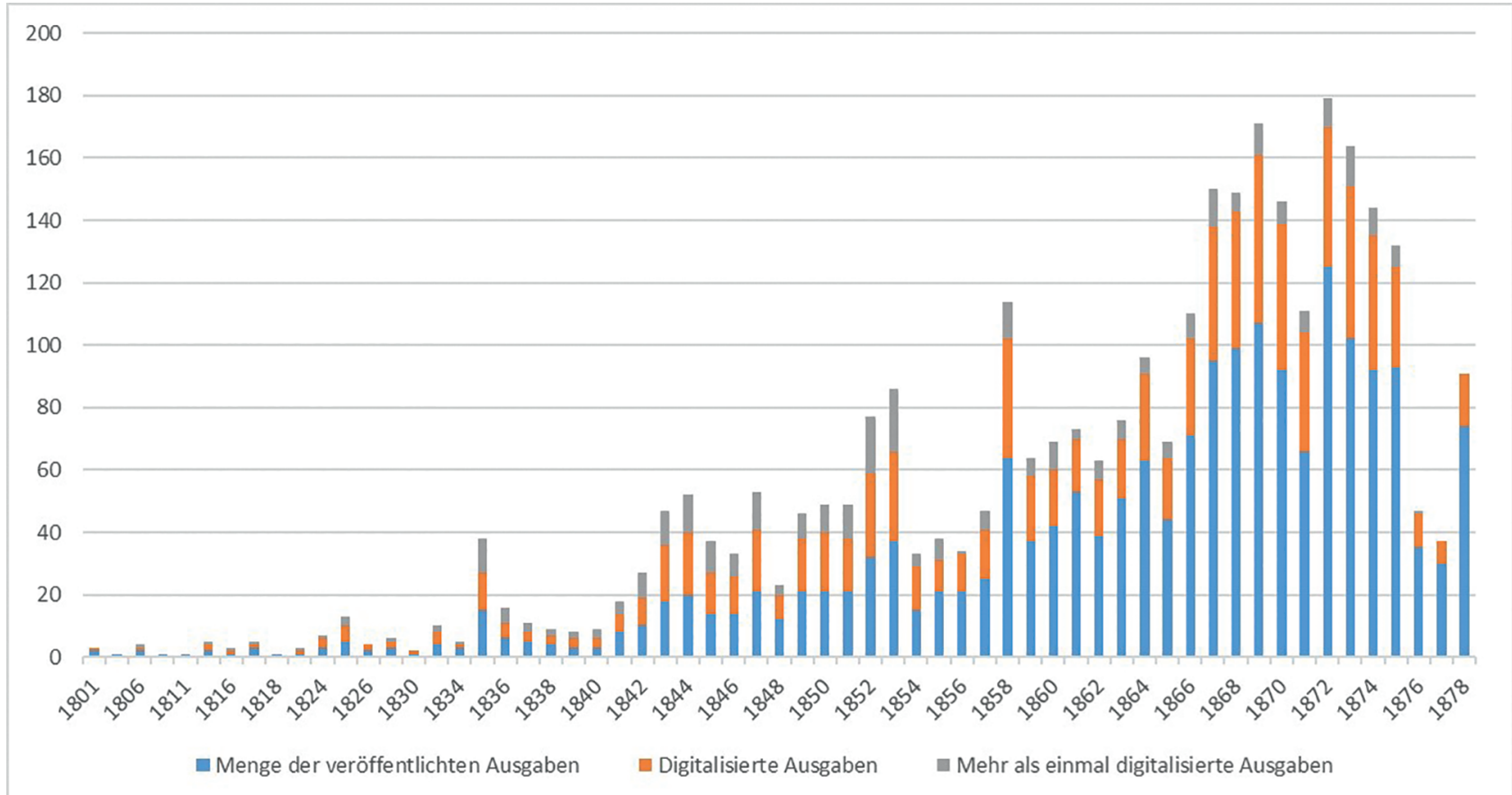

Abb. 2: Anzahl der Veröffentlichungen und Digitalisate nach Jahren

Illustrationen (typografischen Schmuck, Tabellen, Porträts und Karten) nach. Wichtige Zusatzinformationen sind auch die Schriftart (kyrillisch, griechisch, lateinisch, arabisch) und zugehörige Personen wie Mäzene oder Sponsoren.

Alle Elemente der bibliografischen Beschreibung sind durchsuchbar, viele lassen sich als Sortierkriterium verwenden. So ist die geografische Suche nach Sponsor*innen aus einer bestimmten Stadt und eine Auflistung der Bücher, die diese ${ }^{\star}$ r finanziell unterstützt hat, möglich.

Ein thematischer Sucheinstieg in die Bibliografie wird durch die Sacherschließung anhand der Universellen Dezimalklassifikation ermöglicht.

Zusätzlich wird ein geografischer Zugang zur Bibliografie durch Karten und topografische Indizes bereit gestellt, da die Bücher wie oben dargestellt in ganz Europa, aber auch in Istanbul, Moskau oder New York veröffentlicht wurden.

Hier kommt noch einmal die Schwierigkeit der verschiedenen Schreibweisen und Sprachen der Publikationen dieses Zeitraums zum Tragen. Allein das heutige Istanbul ist in den existierenden Bibliografien unter den Namen: Konstantinograd, Konstantinopel, Stambol, Tsare$\operatorname{grad}^{23}$ und Tsarigrad zu finden. Daher wurden Korrelationstabellen sowohl zu Orts- und Personennamen, aber auch zu Titeln angelegt, um eine genaue und einheitliche Abfrage zu ermöglichen.

Ein besonderes Feature der Bibliografie ist z. B. ein JavaScript-basierter Jahreszahlen-Rechner (der sogenannte CyrillicYearConverter), der die kyrillische Zahlschrift ${ }^{24}$ in arabische Ziffern übersetzt. Außerdem wird ein Währungsrechner angeboten, um die damaligen Kosten für bulgarische Bücher umzurechnen, die im Zeitraum von 1801-1878 in verschiedensten Währungen angegeben sind.

\section{Projekt-Abschnitt II: Quantitative und qualitative Analyse der vorhandenen Digitalisate in den verteilten Einrichtungen in Bulgarien}

Von Anfang an wurden in der Bibliografie die digitalisierten Volltexte der bulgarischen Nationalbibliothek „St. St. Cyril and Methodius“ verlinkt. In einem zweiten Schritt sollen digitalisierte Bestände anderer Einrichtungen in Bulgarien hinzugefügt werden.

24 Kyrillische Texte verwendeten damals teilweise Kyrilliza zur Darstellung von Zahlen.

23 Übersetzt: Zarenstadt. 
Bei der Ermittlung von Digitalisaten in den verteilten Einrichtungen Bulgariens und bei der quantitativen Analyse wird deutlich, dass es Dopplungen bei der Digitalisierung gibt, dass aber z. B. aus 23 Jahren des Zeitraums noch weniger als $50 \%$ digitalisiert wurden und dass Publikationen aus vier Jahren des Zeitraums vollständig fehlen.

Bisher fehlt in Bulgarien eine nationale Digitalisierungsstrategie. Entscheidungen, was digitalisiert wird, hängen stark von einzelnen Förderungen ab. Es handelt sich um Einzelentscheidungen der besitzenden Bibliotheken, Museen und Archive. Weiterhin werden die existierenden Digitalisate bisher in verteilten Repositorien bereitgestellt.

Die größte Sammlung liegt bisher in NALIS vor, dem National Academic Library and Information System, das Digitalisate aus verschiedenen Bibliotheken wie z.B. der Universitätsbibliothek „St. Kliment Ohridski“ und der Bibliothek der Akademie der Wissenschaften nachweist. Die Metadaten aus NALIS sind bereits Teil des Semantic Web und damit über Suchmaschinen auffindbar. Darüber hinaus sind die Digitalisate in den jeweiligen Bibliothekskatalogen verlinkt.

Die zweitgrößte Sammlung von Digitalisaten wird von der bulgarischen Nationalbibliothek bereitgestellt. Diese Sammlung war bisher jedoch ausschließlich über den Bibliothekskatalog auffindbar und nicht über Suchmaschinen.

Insgesamt gibt es in Bulgarien eine starke Vielfalt an Software-Lösungen und an Sammlungen, die nicht flächendeckend von Suchmaschinen indexiert werden. Diese Vielfalt erschwert der Forschung den Zugang zu den Digitalisaten und die Arbeit mit dem Material. Teilweise wird die Nutzung der Digitalisate durch nicht-intuitive Nutzerführung der Oberflächen erschwert. Gerade im Hinblick auf seltene Werke und alte Drucke machen fehlende Angaben $\mathrm{zu}$ den Eigenschaften der gedruckten Bücher oder „Korrekturen“ im Digitalisat (das Herausschneiden der Abschnittsheftung, das Entfernen handschriftlicher Einträge, die Auslassung leerer Seiten oder die Korrektur von Paginierungsfehlern) es teilweise weiterhin erforderlich, mit den gedruckten Originalen zu arbeiten. Die Digitalisierung der Literatur dieser seltenen Schriften aus der Wiedergeburtszeit erfordert eine ganz andere Herangehensweise als die Massendigitalisierung standardisiert gedruckter Werke neuerer Zeit. Die qualitative Analyse des vorhandenen Bestands an Digitalisaten zeigt, dass es im Hinblick auf die Sensibilisierung für die Besonderheit der Digitalisierung alter Drucke noch Handlungsbedarf gibt.

\section{Fazit}

Der Mehrwert des vorliegenden Projekts, der Erstellung einer normierten und vollständigen Online-Bibliografie der bulgarischen Wiedergeburtsliteratur, ist für die Forschung $\mathrm{zu}$ dieser geschichtlich bedeutenden Periode in Bulgarien mehr als deutlich. Es ist der vollständige Nachweis der existierenden Werke in einem System und die Auffindbarkeit der Titel über Suchmaschinen.

Auf der anderen Seite ist mit diesem Projekt eine $\mathrm{Zu}$ sammenarbeit zwischen den besitzenden Bibliotheken, Archiven und Museen zustande gekommen. Es wurde ein Grundstein für zukünftige Kooperationen gelegt, um in diesem sehr speziellen Feld, der Digitalisierung seltener Drucke, das hohe Spezialwissen und besondere Kompetenzen zu bündeln.

Die quantitative Analyse der Digitalisate macht darüber hinaus eine strategische Planung für neue Digitalisierungsprojekte möglich, da sie Bulgarienweit bestehende Lücken in der Digitalisierung der Wiedergeburtsliteratur aufzeigt, die in neuen Projekten geschlossen werden können.

\section{Literaturverzeichnis}

Crampton, Richard J. (2007): Bulgaria. New York: Oxford University Press.

Grashkina, Vanja (2007): Vom heiligen Kyrill zum modernen Bibliotheksgesetz. In: BuB : Forum Bibliothek und Information, 59 (1), 53-75.

Härtel, Hans-Joachim; Schönfeld, Roland (1998): Bulgarien: vom Mittelalter bis zur Gegenwart. Regensburg: Pustet.

Jelavich, Charles (1957): Bulgarian Incunabula. In: Library of Congress Quarterly Journal of Current Acquisitions, 14 (3), 77-94.

Teodosiev, Nikolai Milchev (2007): Katalog na bulgarskite petchatni knigi 1508-1878. Sofia: Univ. Isd. Sv. Kliment Ochridski.

Zagorov, Vasil; Encheva, Marina (2017): Digitalization of the Repertoire of Books from the Bulgarian Revival (1801-1878). In: ICERI 2017 Proceedings. 10th International Conference of Education, Research and Innovation, 16-18th November, 2017. Seville: IATED Academy, 3545-48. DOI: 10.21125/iceri.2017.0961.

Zlatkova, Plamena; Angelova, Gabriela (2019): The Bulgarian libraries and their experience in digitizing rare books and special collections. In: ICERI 2019 Proceedings. 12th International Conference of Education, Research and Innovation, 11-13th November, 2019. Seville: IATED Academy, 3864-71. DOI: 10.21125/iceri.2019. 0980. 


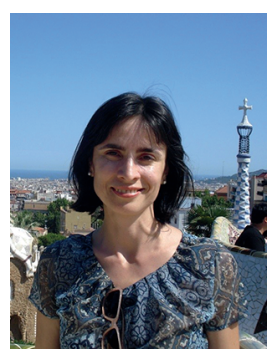

\section{Marina Encheva}

University of Library Studies and

Information Technologies

Library Sciences

119 Tsarigradsko Chausee

BG-1784 Sofia

Bulgarien

m.encheva@unibit.bg

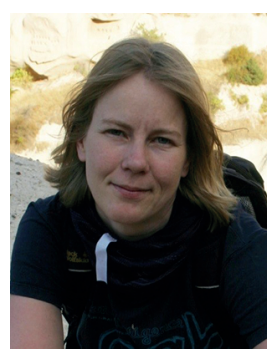

\section{Nicole Krüger}

ZHAW - Zürcher Hochschule für Angewandte Wissenschaften

Hochschulbibliothek Winterthur

Turbinenstrasse 2

Postfach

$\mathrm{CH}-8401$ Winterthur

Schweiz

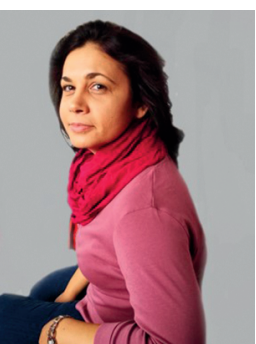

\section{Plamena Zlatkova}

University of Library Studies and Information Technologies

119 Tsarigradsko Chausee

BG-1784 Sofia

Bulgarien

p.zlatkova@unibit.bg

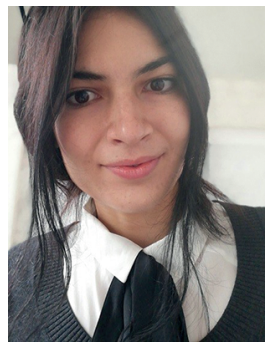

\section{Gabriela Angelova}

University of Library Studies and Information Technologies Library Sciences

119 Tsarigradsko Chausee

BG-1784 Sofia

Bulgarien

g.angelova@unibit.bg

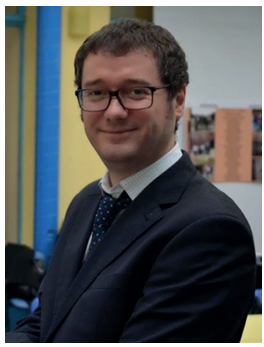

\section{Vasil Zagorov}

University of Library Studies and

Information Technologies

Library Sciences

119 Tsarigradsko Chausee

BG-1784 Sofia

Bulgarien

v.zagorov@unibit.bg 